\title{
Reduced Intellectual Ability in Offspring Born from Preeclamptic Mothers: A Prospective Cohort Study
}

This article was published in the following Dove Press journal: Risk Management and Healthcare Policy

\author{
Linli Liu' \\ Zhou Lin' \\ Beihong Zheng ${ }^{2}$ \\ Lanlan Wang' \\ Jianqin Zou' \\ Sanshan $\mathrm{Wu}^{\prime}$ \\ Zhongqing Jiang ${ }^{\prime}$ \\ Qiong Jin ${ }^{1}$ \\ Xuedan Lai ${ }^{1}$ \\ Peihong Lin' \\ 'Department of Gynecology and \\ Obstetrics, Fuzhou First Hospital \\ Affiliated to Fujian Medical University, \\ Fuzhou 350004, People's Republic of \\ China; ${ }^{2}$ Center for Assisted \\ Reproductive Technology, Fujian \\ Provincial Maternity and Children's \\ Hospital, Affiliated Hospital of Fujian \\ Medical University, Fuzhou 350004, \\ People's Republic of China
}

Background: Severe preeclampsia may affect placental development, and high homocysteine (Hcy) levels are linked to intellectual disability. However, the correlation between perinatal Hcy levels and intellectual ability remains unknown in severe preeclampsia-affected offspring. Objective: We aimed to investigate the intellectual ability in offspring born from preeclamptic mothers and examine the role of prenatal Hcy in the prediction of intellectual disability in preschool-aged offspring.

Methods: The IQ scores were compared between 101 children born to mothers with severe preeclampsia and 202 offsprings born to normotensive mothers. Maternal Hcy levels within 7 days prior to delivery and postnatal cord blood Hcy were measured. The associations of Hcy with IQ scores were evaluated, and the optimal cut-off values for predicting intellectual disability in the offspring were estimated.

Results: The children born to mothers with severe preeclampsia had a greater postnatal cord blood Hcy than those born from normotensive mothers $(P<0.001)$, and the mothers with severe preeclampsia presented a higher prenatal Hcy $(P<0.001)$. The children born to mothers with severe preeclampsia had significantly lower IQ scores than those born from normotensive mothers, and a higher Hcy was associated with a lower IQ in preeclampsiaaffected offspring. The prevalence of intellectual disability was 2.86 times higher in severe preeclampsia-affected offspring than in children born from normotensive mothers, and the prevalence of low IQ was greater in children born to mothers with severe preeclampsia than in those from normotensive mothers. ROC curve analysis showed that both maternal and cord blood Hcy were predictors of intellectual disability, and the optimal cut-off for predicting intellectual disability was 17.7 and $9.75 \mu \mathrm{mol} / \mathrm{L}$ for maternal and cord blood Hcy.

Conclusion: Perinatal exposure to severe preeclampsia has an adverse effect on postnatal intellectual development, and high maternal and cord blood Hcy may contribute to this association.

Keywords: preeclampsia, homocysteine, intellectual ability, ROC curve analysis

\section{Introduction}

Preeclampsia, a condition characterized by high blood pressure in pregnant women, is estimated to affect $7.5 \%$ of the pregnancies worldwide. ${ }^{1-4}$ This disorder has been found to adversely affect fetal brain development due to hypoxia, 5,6 and severe preeclampsiaaffected offspring may present poorer neurodevelopmental functions, relative to those born from normotensive mothers, such as reduced cognitive performance ${ }^{7,8}$ and verbal ability. $^{9-14}$ However, the effect of severe preeclampsia on the development of intelligence in the offspring has not been fully demonstrated until now.
Correspondence: Linli Liu Department of Gynecology and Obstetrics, Fuzhou First Hospital Affiliated to Fujian Medical University, Fuzhou City, Fujian Province 350004,

People's Republic of China

Tel/Fax +86-59l-88301693

Email applewang814@I63.com
Risk Management and Healthcare Policy 2020:I3 2037-2046

2037

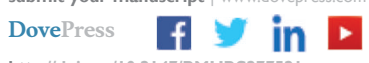


Previous studies have shown that severe preeclampsia is associated with endothelial dysfunction ${ }^{15}$ and may affect placental development, ${ }^{16}$ which results in reduced blood flow to the fetus and a relatively hypoxic state. ${ }^{17}$ Homocysteine (Hcy) is an endogenous sulphydryl-containing amino acid produced by transmethylation of the essential sulfur-containing amino acid, methionine. ${ }^{18}$ High Hcy levels have been reported to cause endothelial dysfunction, ${ }^{19,20}$ a known risk factor for intellectual disability and hypoxic damage. ${ }^{21,22}$ These pathophysiological changes may affect neurodevelopmental functions as early as the fetal stage. ${ }^{23,24}$ To the best of our knowledge, however, the correlation between perinatal Hcy levels and intellectual ability remains unknown in severe preeclampsia-affected offspring. This prospective cohort study was therefore designed to investigate the intellectual ability in offspring born from preeclamptic mothers and examine the role of prenatal Hcy in the prediction of intellectual disability in preschool-aged offspring.

\section{Methods}

\section{Study Subjects}

A single-center prospective cohort study was designed with aims to compare the intellectual ability in children born to singleton pregnant women with and without severe preeclampsia delivered in the Department of Gynecology and Obstetrics, Fuzhou First Hospital (Fuzhou, China), during the period between January 2010 and December 2015. Severe preeclampsia was diagnosed based on at least one of the following criteria: ${ }^{25}$ systolic blood pressure $\geq 160 \mathrm{mmHg}$ or diastolic blood pressure $\geq 110 \mathrm{mmHg}$ on two occasions separated by at least $6 \mathrm{~h}$ after 20 weeks of gestation; proteinuria $\geq 5 \mathrm{~g}$ in $24 \mathrm{~h}$ or $\geq 3 \mathrm{~g}$ on a dipstick; oliguria ( $\leq 500 \mathrm{~mL}$ in $24 \mathrm{~h}$ ); cerebral or visual disturbances; epigastric pain; thrombocytopenia; elevated serum liver enzymes; pulmonary oedema or cyanosis; uteroplacental dysfunction; and fetal growth restriction or eclampsia. Those who met the following criteria were excluded from the study: missing data, alcohol/drug/smoking abuse in one of the parents during or before pregnancy, mild preeclampsia or gestational hypertension, chronic hypertension (hypertension requiring treatment with antihypertensive agents before the relevant pregnancy), pre-gestational diabetes, renal diseases, systemic lupus erythematosus, multiple pregnancies, neonatal and fetal death, or major fetal congenital malformations.

A total of 172 mothers/children pairs that met the inclusion and exclusion criteria were recruited, and 101 pairs that agreed to participate in a follow-up until the children's age of 4 years were included in the study (Figure 1). For each pair, we enrolled two controls matched by gestational age (within 1 week), birth weight (within $100 \mathrm{~g}$ ), and current age of children (within 6 months). For control subjects, singleton pregnant women with normal blood pressure (systolic blood pressure $<140 \mathrm{mmHg}$ and diastolic blood pressure $<90$ $\mathrm{mmHg}$ ) during pregnancy were included, and those complicated by internal or surgical diseases, gestational diabetes, thyroid diseases or mental disorders were excluded from this study. Both preeclampsia patients and normotensive controls were recruited in this study via telephone follow-up by two well-trained senior obstetricians during the period between January 2014 and December 2019. Medical records were carefully reviewed and parental interviews were performed by two well-trained senior obstetricians to capture parental demographics, perinatal data, birth outcomes, and postnatal factors. In this study, the parental education level was categorized as (middle school or below), middle (high school), and high (college or above).

\section{Measurement of Prenatal and Postnatal Hcy}

Maternal Hcy levels within 7 days prior to delivery and postnatal cord blood Hcy levels were measured using enzymelinked immunosorbent assay (ELISA) with a commercial kit (Senbeijia Biotechnology Co., Ltd.; Nanjing, China).

\section{Assessment of Intellectual Ability in the Offspring}

The Chinese Wechsler Young Children Scale of Intelligence (C-WYCSI) was employed to assess the intellectual ability in the pre-school children at the ages of 4 years. ${ }^{26}$ The C-WYCSI is designed for assessment of intelligence in children at ages of 4 to 6 years and consists of five verbal subtests (arithmetic, information, comprehension, sorting, and vocabulary) and five performance subtests (picture completion, animal pegs, block design, object assembly, and mazes). ${ }^{26}$ All subtests use a scaled scoring system, which is standardized using a mean of 10 and a standard deviation of 3 to transform the raw score. In addition, the verbal intelligence quotient (VIQ) and performance intelligence quotient (PIQ) scores were calculated with their sum representing the full intelligence quotient (FIQ) score. All intelligence tests were performed by two welltrained psychologists that were blinded to the study.

\section{Ethics Consideration}

This study was approved by the Ethics Review Committee of Fuzhou First Hospital (date of approval: December 5, 


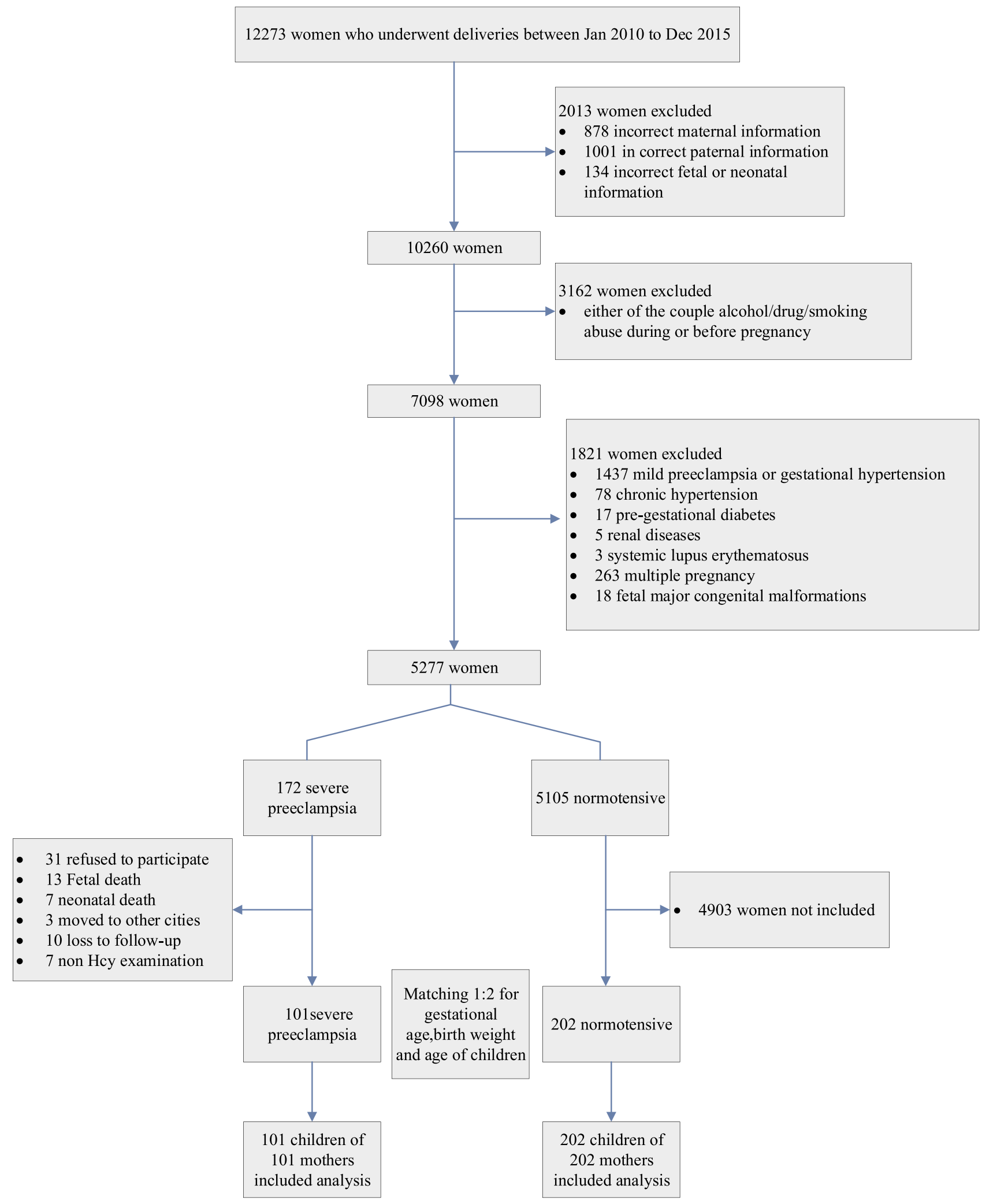

Figure I Flow chart of study subjects selection.

2017; approval no.: FZSY-201,700,854). All procedures were performed following the Declaration of Helsinki, as well as international and national laws, guidelines and regulations. Signed informed consent was obtained from all subjects with a detailed description of the purpose of the study. 


\section{Statistical Analysis}

We analyzed the data distribution using the KolmogorovSmirnov and Shapiro-Wilk tests. All measurement data were expressed as mean \pm standard deviation (SD) or median (interquartile range) as appropriate and were compared using the Student's $t$-test or Mann-Whitney $U$-test, while all categorical data were described as a number (percentage) and were compared using chisquare test or Fisher's exact test. The mean differences in FIQ, VIQ, and PIQ scores were calculated using a univariate general linear regression model after adjusting for confounding factors, and the associations of Hcy levels with IQ scores were examined using the Pearson correlation analysis and logistic regression analysis. The optimal cut-off values for predicting intellectual disability in the offspring were estimated with a receiver operating characteristic (ROC) curve analysis. All statistical analyses were conducted using the statistical software SPSS version 19.0 (SPSS, Inc.; Chicago, IL, USA), and a $P$ value of $<0.05$ was indicative of statistical significance.

\section{Results}

\section{Cohort Characteristics}

A total of 12,273 women underwent deliveries in the Department of Gynecology and Obstetrics, Fuzhou First Hospital during the period between January 2010 and December 2015, and finally, 101 severe preeclampsia-affected offspring and 202 children born from normotensive mothers were enrolled in this study according to the inclusion and exclusion criteria (Figure 1). Tables 1 and 2 present the study subjects' characteristics. The children born to mothers

Table I Children and Perinatal Baseline Characteristics

\begin{tabular}{|c|c|c|c|}
\hline Characteristic & $\begin{array}{l}\text { Severe Preeclampsia-Affected } \\
\text { Offspring }(n=10 I)\end{array}$ & $\begin{array}{l}\text { Offspring Born from } \\
\text { Normotensive Mothers }(n=202)\end{array}$ & $P$ value \\
\hline Age (year) & $4.16 \pm 0.17$ & $4.16 \pm 0.31$ & 0.81 \\
\hline Male (N, \%) & $44(43.56)$ & $108(53.46)$ & 0.066 \\
\hline BMI $\left(\mathrm{kg} / \mathrm{m}^{2}\right)$ & $16.32 \pm 1.26$ & $16.62 \pm 1.48$ & 0.083 \\
\hline Hcy level ( $\mu \mathrm{mol} / \mathrm{L})$ & $5.55 \pm 1.78$ & $5.18 \pm 1.24$ & 0.292 \\
\hline \multicolumn{4}{|l|}{ Gestational complications } \\
\hline GDM (N, \%) & $29(28.71)$ & $37(18.32)$ & 0.039 \\
\hline Pregnancy with thyroid dysfunction $(\mathrm{N}, \%)$ & $5(4.95)$ & $12(5.94)$ & 0.724 \\
\hline $\mathrm{ICP}(\mathrm{N}, \%)$ & $0(0)$ & I $(0.50)$ & 0.999 \\
\hline Placenta previa $(\mathrm{N}, \%)$ & $3(2.97)$ & $5(2.48)$ & 0.802 \\
\hline Placental abruption (N, \%) & $2(1.98)$ & $\mathrm{I}(0.5)$ & 0.259 \\
\hline PROM (N, \%) & $9(8.91)$ & $32(15.84)$ & 0.138 \\
\hline NEUC (coils, cm) & $0(0,1)$ & $0(0,1)$ & 0.058 \\
\hline GBS (N, \%) & $3(2.97)$ & $7(3.47)$ & 0.819 \\
\hline Post-delivery cord blood Hcy ( $\mu \mathrm{mol} / \mathrm{L})$ & $11.6 \pm 3.28$ & $6.45 \pm 2.75$ & $<0.001$ \\
\hline \multicolumn{4}{|l|}{ Delivery data } \\
\hline Labor time $(\mathrm{h})$ & $6.36 \pm 4.36$ & $6.77 \pm 4.67$ & 0.462 \\
\hline Birth weight $(g)$ & $2824.93 \pm 775.90$ & $2867.79 \pm 777.04$ & 0.651 \\
\hline Gestational age at delivery (weeks) & $37.37 \pm 2.51$ & $37.48 \pm 2.55$ & 0.725 \\
\hline Cesarean section $(\mathrm{N}, \%)$ & $65(65.36)$ & $108(53.46)$ & 0.071 \\
\hline Apgar score $<7$ at $5 \min (\mathrm{N}, \%)$ & $8(7.92)$ & $7(3.47)$ & 0.053 \\
\hline Respiratory distress syndrome (N, \%) & $3(2.97)$ & I (0.49) & 0.259 \\
\hline Assisted ventilation & II (I0.89) & II (5.45) & 0.085 \\
\hline Admission to NICU & $33(32.67)$ & $46(22.77)$ & 0.064 \\
\hline Turbid amniotic fluid, (N, \%) & $17(16.83)$ & $19(9.4 I)$ & 0.683 \\
\hline Breastfeeding (month) & $10(5.5,12)$ & $10(6,13)$ & 0.772 \\
\hline
\end{tabular}

Notes: Data are described as mean \pm standard deviation, number (percent) or median (interquartile range). $P$ values are calculated by independent Student's $t$-test, MannWhitney $U$-test, chi-square test or Fisher's exact test.

Abbreviations: PE, preeclampsia; BMI, body mass index; Hcy, homocysteine; GDM, gestational diabetes; ICP, Intrahepatic cholestasis of pregnancy; PROM, premature rupture of fetal membranes; NEUC, umbilical cord around the neck; GBS, group B streptococcal. 
Table 2 Parental Baseline Demographic and Clinical Characteristics

\begin{tabular}{|c|c|c|c|}
\hline Characteristic & Mothers with Severe PE $(n=101)$ & Normotensive Mothers $(n=202)$ & $P$ value \\
\hline \multicolumn{4}{|l|}{ Maternal factors } \\
\hline Maternal age (year) & $29.58 \pm 4.74$ & $28.80 \pm 4.85$ & 0.181 \\
\hline Gravidity & $2(2,4)$ & $2(1,3)$ & 0.477 \\
\hline Parity & $2(1,3)$ & $2(1,2)$ & 0.316 \\
\hline BMI at delivery $\left(\mathrm{kg} / \mathrm{m}^{2}\right)$ & $23.61 \pm 1.85$ & $24.35 \pm 2.05$ & 0.711 \\
\hline Pre-delivery maternal Hcy $(\mu \mathrm{mol} / \mathrm{L})$ & $17.42 \pm 6.67$ & $6.53 \pm 1.79$ & $<0.001$ \\
\hline \multicolumn{4}{|l|}{ Maternal education level } \\
\hline Low (N, \%) & 21 (20.79) & $68(33.66)$ & 0.064 \\
\hline Middle (N, \%) & $45(44.55)$ & $62(30.69)$ & \\
\hline $\operatorname{High}(\mathrm{N}, \%)$ & $35(34.65)$ & $72(35.64)$ & \\
\hline Hemoglobin $(g / L)$ & $96.66 \pm 9.75$ & $96.1 \pm 9.59$ & 0.635 \\
\hline Cholesterol (mmol/L) & $4.35(3.97,5.21)$ & $2.3(1.86,4.52)$ & $<0.001$ \\
\hline HDL-C ( $\mu \mathrm{mmol} / \mathrm{L})$ & $1.43(1.22,1.93)$ & $1.87(0.9,2.56)$ & 0.908 \\
\hline LDL-C (mmol/L) & $2.14(1.79,3.03)$ & $1.9(1.23,3.46)$ & 0.3 \\
\hline Paternal age (year) & $30.63 \pm 4.7$ & $30.25 \pm 5.2$ & 0.534 \\
\hline \multicolumn{4}{|l|}{ Paternal education level } \\
\hline Low (N, \%) & $18(17.82)$ & $34(16.83)$ & 0.976 \\
\hline Middle (N, \%) & $62(61.39)$ & $126(62.38)$ & \\
\hline High (N, \%) & 21 (20.79) & $42(20.79)$ & \\
\hline
\end{tabular}

Notes: Data are expressed as mean \pm standard deviation, number (percent) or median (interquartile range). $P$ values are calculated by independent Student's $t$-test, MannWhitney U-test, chi-square test or Fisher's exact test.

Abbreviations: PE, preeclampsia; BMI, body mass index; Hcy, homocysteine; HDL, high-density lipoprotein cholesterol; LDL, Low-density lipoprotein cholesterol.

with severe preeclampsia had a greater postnatal cord blood Hcy concentration than those born from normotensive mothers $(P<0.001)$, and the mothers with severe preeclampsia presented a higher prenatal maternal Hcy level $(P<0.001)$ and maternal serum cholesterol $(P<0.001)$, and were more likely to be affected by gestational diabetes mellitus $(P=0.039)$. No between-group differences were observed in terms of other perinatal and parental characteristics $(P>0.05)$. Therefore, these variables were excluded as potential confounders to maintain a steadier model.

\section{A Lower IQ Score is Measured in Severe Preeclampsia-Affected Offspring}

Compared to the control group (unadjusted), children exposed to severe preeclampsia exhibited lower VIQ (11.39, 95\% CI: 8.68-14.09), PIQ (10.32, 95\% CI: 8.0612.59), and FIQ scores (5.83, 95\% CI: 3.44-8.21), and adjustment for maternal cholesterol and gestational diabetes mellitus in the general linear regression model did not attenuate this association. However, the differences were insignificant after adjusting for postnatal cord blood Hcy in model 3 (FIQ $=1.22,95 \%$ CI: $-4.21-1.77$ ) and maternal Hcy in model 4 (FIQ $=2.62,95 \%$ CI: -0.98 to 6.21) (Table 3).

\section{A Higher Hcy Level is Associated with a Lower IQ Score}

To further investigate the effect of high Hcy levels on intellectual disability in children born from mothers with severe preeclampsia, the case and control groups were further divided into the high- and low-Hcy subgroups according to the 75th percentile of the maternal serum Hcy level at delivery, and the mean maternal Hcy concentrations were $14.21 \mu \mathrm{mol} / \mathrm{L}$ in the low-Hcy case subgroup, $27.32 \mu \mathrm{mol} / \mathrm{L}$ in the high-Hcy case subgroup, $5.90 \mu \mathrm{mol} / \mathrm{L}$ in the low-Hcy control subgroup and $8.84 \mu \mathrm{mol} / \mathrm{L}$ in the high-Hcy control subgroup, respectively. In addition, a higher FIQ score was measured in the low-Hcy control subgroup than in both the high- and low-Hcy case subgroups, and a lower FIQ score was found in the high-Hcy case subgroup than in the low-Hcy case and control subgroups. In addition, there was no significant association between the high- and low-Hcy control subgroups (Figure 2A), and similar results were observed for subgroups based on the postnatal cord blood Hcy (Figure 2B). 


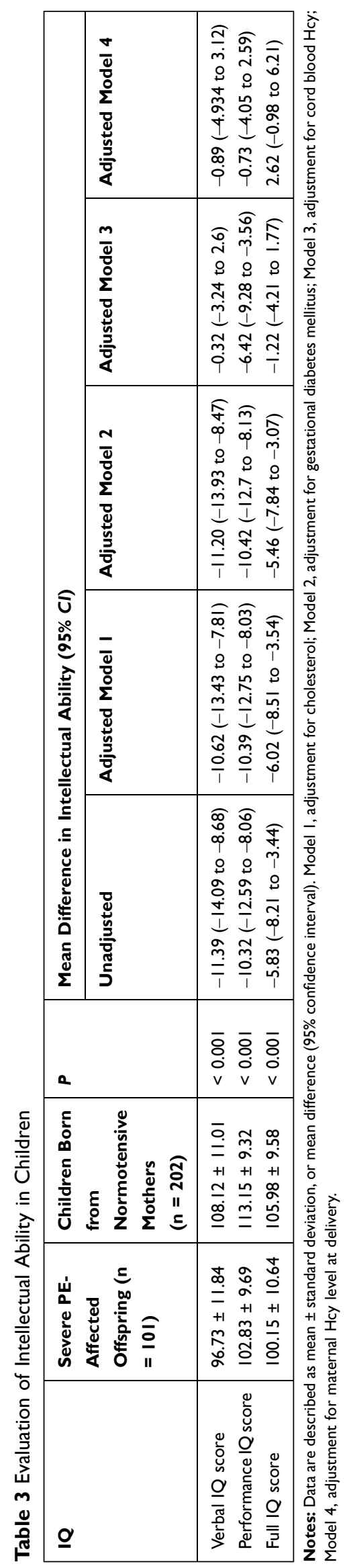

\section{Higher Prevalence of Intellectual Disability in Severe Preeclampsia-Affected Offspring}

We investigated the prevalence of intellectual disability $(\mathrm{IQ}<80)$ in both groups, and the prevalence of intellectual disability was 2.86 times higher in the severe preeclampsia-affected offspring than in children born from normotensive mothers, and the prevalence of low VIQ, PIQ and FIQ were all greater in children born to mothers with severe preeclampsia than in those from normotensive mothers (Table 4).

\section{Prenatal Hcy Contributes to Intellectual Disability in Severe Preeclampsia-Affected Offspring}

Linear correlation analysis revealed negative associations of maternal $(r=-0.404, P<0.001)$ and cord blood Hcy $(r=-0.367, P<0.001)$ with FIQ score in a pooled group $(\mathrm{n}=303)$. Then, the case group was further divided into the intellectual disability $(n=20)$ and non-intellectual disability $(\mathrm{n}=81)$ subgroups based on the IQ scores (a score of $<80$ in one or more of the IQ tests was defined as intellectual disability), and a higher rate of gestational diabetes mellitus, a higher proportion of caesarean section, higher levels of maternal and cord blood Hcy, a lower gestational age at delivery, and lower birth weight were found in the intellectual disability subgroup than in the non-intellectual disability subgroup $(P<0.05)$. These factors were therefore included in the logistic regression analysis. After adjusting for potential confounders, multivariate logistic regression analysis showed that the maternal and cord blood Hcy were independent risk factors for intellectual disability with an adjusted relative risk of 0.82 (95\% CI: $0.67-0.99)$ and 0.68 (95\% CI: 0.54-0.86), respectively (Table 5). ROC curve analysis showed that both maternal (area under the curve $=0.87,95 \%$ CI: 0.79 -0.94 ) and cord blood Hcy (area under the curve $=0.91$, 95\% CI: 0.85-0.95) were predictors of intellectual disability (Figure 3), and the optimal cut-off for predicting intellectual disability was 17.7 and $9.75 \mu \mathrm{mol} / \mathrm{L}$ for maternal and cord blood Hcy, which exhibited a satisfactory sensitivity (Table 6).

\section{Discussion}

In this study, we found a significantly higher prevalence rate of intelligence ability in severe preeclampsia-affected 
A

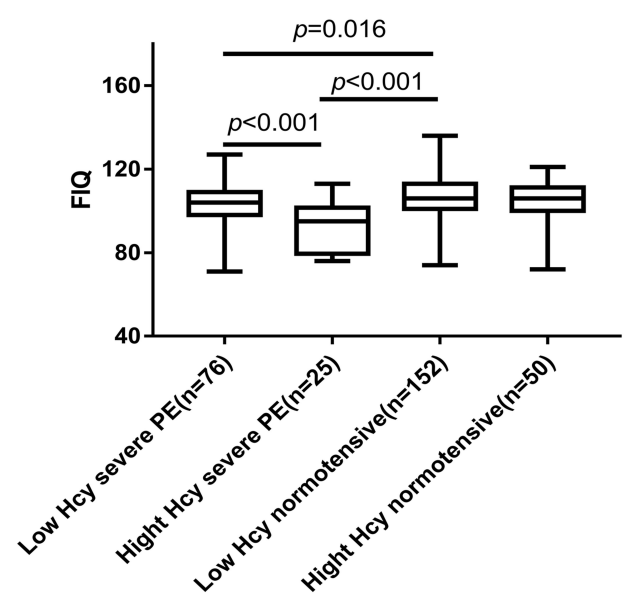

B

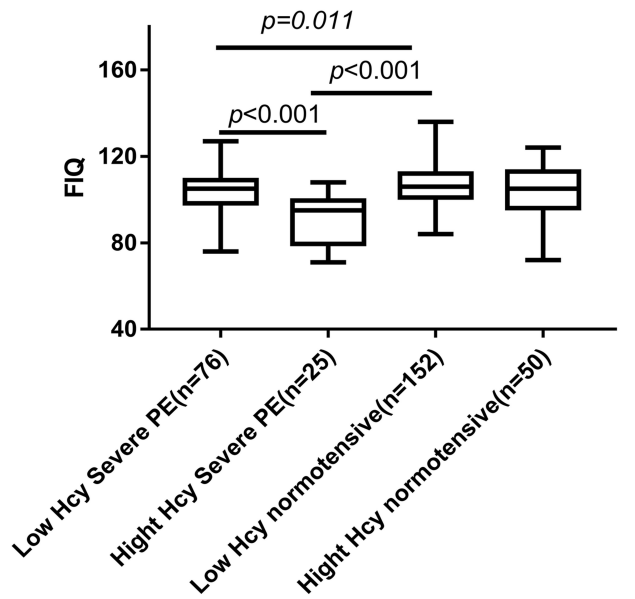

Figure 2 Association of Hcy levels with intelligence quotient scores. (A) Higher maternal serum Hcy levels are associated with lower intelligence quotients. The boxes extend from the 25 th to 75 th percentiles, and the whiskers indicate the 5 th and 95 th percentiles. The lines in the middle of the boxes are plotted at the median value. (B) Higher cord blood Hcy levels are associated with lower intelligence quotient scores. The boxes extend from the 25 th to 75 th percentiles, and the whiskers indicate the 5 th and 95th percentiles. The lines in the middle of the boxes are plotted at the median value.

Abbreviations: PE, preeclampsia; FIQ, full intelligence quotient.

offspring than in children born from normotensive mothers at the ages of 4 years. To the best of our knowledge, this is the first study to report a strong association between increased maternal and cord blood Hcy levels and intellectual disability in children born to mothers with severe preeclampsia.

Preeclampsia is a pregnancy-specific complication influenced by a number of factors. ${ }^{27,28}$ Among numerous pathological changes involved in preeclampsia, the most important

Table 4 Prevalence of Intellectual Disability in the Offspring

\begin{tabular}{|l|l|l|l|}
\hline IQ & $\begin{array}{l}\text { Severe } \\
\text { Preeclampsia- } \\
\text { Affected } \\
\text { Offspring } \\
\text { (n= I0I) }\end{array}$ & $\begin{array}{l}\text { Offspring Born } \\
\text { from } \\
\text { Normotensive } \\
\text { Mothers } \\
(\mathbf{n}=\mathbf{2 0 2})\end{array}$ & P value \\
\hline Verbal IQ & $\begin{array}{l}\text { I4 (13.86\%) } \\
6(5.94 \%)\end{array}$ & $\begin{array}{l}8(3.96 \%) \\
0(0.00 \%)\end{array}$ & 0.002 \\
Performance IQ & $\begin{array}{l}(8.91 \%) \\
\text { Full IQ }\end{array}$ & $\begin{array}{l}(2.48 \%) \\
0.015\end{array}$ \\
\hline
\end{tabular}

Notes: Intellectual disability is defined as an IQ score of $<80$. Data are shown as $N(\%)$. $P$ values were calculated using the chi-square test or Fisher's exact test. are endothelial dysfunction, local inflammation, and oxidative stress. $^{29-31}$ Previous studies have shown that increased Hcy levels augment cellular oxidative stress and cause generalized endothelial dysfunction, ${ }^{32-34}$ and increased plasma oxidative stress has been reported in mothers with preeclampsia. $^{35}$ In addition, elevated Hcy levels appear to impair endothelium and lead to ischemic hypoxia through impaired nitric oxide-dependent vasodilation, endothelial toxicity and injury, oxidative stress, and systemic inflammation. ${ }^{36-40}$ A recent study reported a significant association of serum Hcy concentration with the severity of the intellectual disability, ${ }^{41}$ and increased plasma Hcy levels have also been identified as an independent risk factor for cognitive decline and atrophic changes in the brain. ${ }^{42-44}$ It is therefore hypothesized that mothers with preeclampsia may present endothelial dysfunction induced by elevated Hcy levels, which results in hypoxic damages and leads to subsequent adverse effects on fetal neurodevelopment.

In this study, the offspring of normotensive mothers had greater intellectual performance scores than those born to mothers with severe preeclampsia at age of

Table 5 Multivariate Logistic Regression Analysis of Risk Factors for Intellectual Disability in Severe Preeclampsia-Affected Offspring

\begin{tabular}{|l|l|l|l|l|}
\hline Factor & RR (95\% Cl) & P & aRR (95\% Cl) \\
\hline Pre-delivery maternal Hcy & $0.89(0.69-0.99)$ & 0.044 & $0.82(0.67-0.99)$ & 0.043 \\
Post-delivery cord blood Hcy & $0.67(0.52-0.84)$ & 0.001 & $0.68(0.54-0.86)$ & 0.001 \\
\hline
\end{tabular}

Notes: The model is adjusted for gestational diabetes, cesarean section, pre-delivery maternal serum Hcy, post-delivery cord blood Hcy, gestational age at delivery and birth weight.

Abbreviations: $\mathrm{RR}$, relative risk; aRR, adjusted relative risk; $\mathrm{Cl}$, confidence interval. 


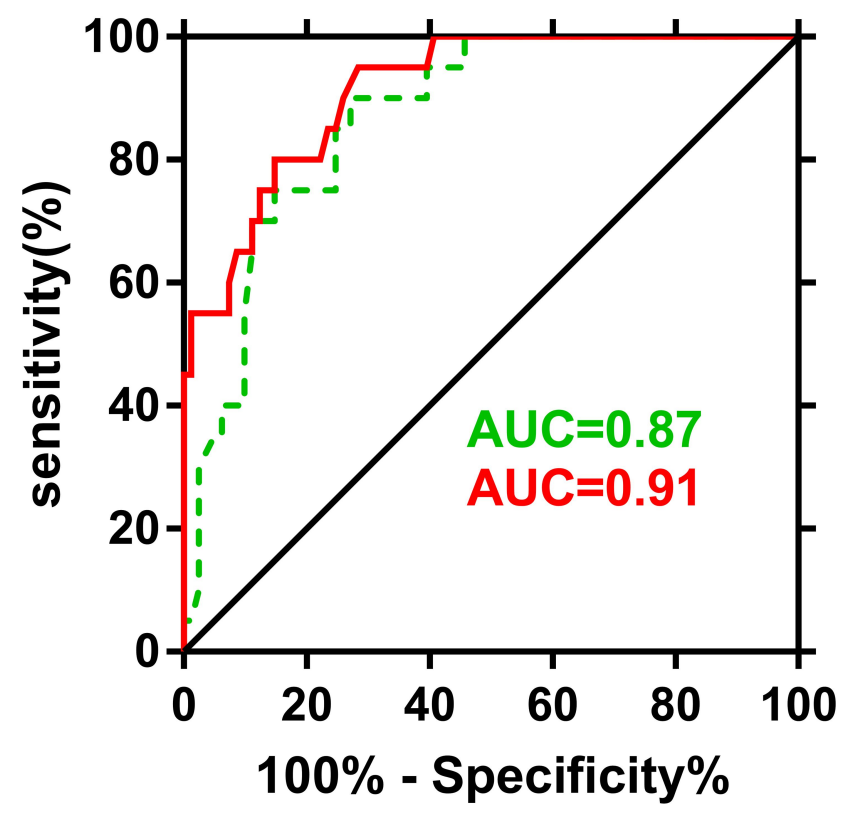

Figure 3 ROC curve analysis of maternal and cord blood Hcy levels for prediction of intellectual disability.

4 years, which was consistent with previous studies reporting the development of impaired IQ in severe preeclampsia-affected offspring as early as childhood. ${ }^{10-14}$ Preeclampsia has been identified as an adverse risk factor for impaired cognitive development in children; ${ }^{7}$ however, there is also evidence showing no association between preeclampsia impaired cognitive development among infants, 9-15-year-old children, and adults. ${ }^{11,45,46}$ Unlike previous studies, the present study enrolled participants with severe preeclampsia without hypertension or mild preeclampsia, in line with the previously reported direct association between Hcy and severe preeclampsia but not hypertension or mild preeclampsia. ${ }^{47}$ Another distinctive feature of our study is that we matched covariates such as birth weight, gestational age, and current age of children, which improved the between-group estimation of the association between severe preeclampsia and childhood IQ. This supports our findings that prenatal exposure to severe preeclampsia reduces intellectual ability in the offspring.
Notably, we observed no significant differences in the IQ scores after adjusting for maternal and cord blood Hcy levels in models 3 and 4, respectively, indicating that prenatal Hcy levels may affect intelligence ability. Then, the participants were further divided into the high- and low-Hcy subgroups according to the 75th percentile of the maternal serum Hcy level at delivery, and a reduced FIQ score was measured in the high-Hcy case subgroup, which had mean maternal and cord blood Hcy levels of 27.32 and $15.55 \mu \mathrm{mmol} / \mathrm{L}$, respectively. In addition, linear correlation analysis demonstrated a negative effect of Hcy levels on offspring IQ. We divided the case group into two subgroups based on the IQ score, and logistic regression analysis identified maternal and cord blood Hcy levels as independent risk factors for predicting intellectual disability. These findings indicate a strong value of increased Hcy levels for predicting adverse neurological functions. ROC curve analysis revealed the optimal maternal and cord blood Hcy cut-off of 17.7 and $9.75 \mu \mathrm{mmol} / \mathrm{L}$ for intellectual disability, respectively. These data suggest that prenatal Hcy level may be a predisposing factor affecting intellectual development in severe preeclampsia-affected offspring. However, the maternal gestational Hcy levels, as well as other biomarkers including glutathione peroxidase, catalase, malondialdehyde, superoxide dismutase, and nitric oxide synthase, which may affect offspring IQ, were not measured. Further studies are required to investigate the impact of other potential factors on intellectual development in severe preeclampsia-affected offspring.

This study has several limitations. First, all participants were recruited from a single center. Second, we only evaluated the intelligence levels at a single time point (at 4 years of age) rather than at multiple time points.

In summary, the results of the present study demonstrate that perinatal exposure to severe preeclampsia has an adverse effect on postnatal intellectual development, and high maternal and cord blood Hcy levels may contribute to this association. Our data provide insights into the management of intellectual disability in children born from mothers with severe preeclampsia.

Table 6 ROC Curve Analysis of Maternal and Cord Blood Hcy for Prediction of Intellectual Disability

\begin{tabular}{|l|l|l|l|l|l|l|}
\hline Hcy Level & AUC (95\% Cl) & Cutoff & $\begin{array}{l}\text { Maximum } \\
\text { Youden's Index }\end{array}$ & Sensitivity (\%) & Specificity (\%) & P value \\
\hline Pre-delivery maternal Hcy $(\mu \mathrm{mol} / \mathrm{L})$ & $0.87(0.79-0.94)$ & 17.7 & 0.628 & 90 & 72.8 & $<0.001$ \\
Post-delivery cord blood Hcy $(\mu \mathrm{mol} / \mathrm{L})$ & $0.91(0.85-0.95)$ & 9.75 & 0.321 & 100 & 32.1 & $<0.001$ \\
\hline
\end{tabular}

Abbreviation: AUC, area under the curve. 


\section{Funding}

This study was funded by the grants from the Department of Science and Technology of Fuzhou City (grant nos. 2018-S-105-4 and 2017-S-129-3), the Construction Project for Clinical Medicine Center in Fujian Province (grant no. 201610192), and the Natural Science Foundation of Fujian Province (grant no. 2019D004).

\section{Disclosure}

The authors declare no relevant financial, personal, political, intellectual or religious conflicts of interests for this work.

\section{References}

1. Abalos E, Cuesta C, Grosso AL, Chou D, Say L. Global and regional estimates of preeclampsia and eclampsia: a systematic review. Eur J Obstet Gynecol Reprod Biol. 2013;170:1-7. doi:10.1016/j.ejogrb. 2013.05.005

2. Eiriksdottir VH, Valdimarsdottir UA, Asgeirsdottir TL, et al. Pregnancy-induced hypertensive disorders before and after a national economic collapse: a population based cohort study. PLoS One. 2015;10:e0138534.

3. Ozeki A, Tani K, Takahashi H, et al. Preeclamptic patient-derived circulating cell-free DNA activates the production of inflammatory cytokines via toll-like receptor 9 signalling in the human placenta. J Hypertens. 2019;37:2452-2460. doi:10.1097/HJH.000000000 0002208

4. Lv LJ, Wu LL, Wen JY, et al. Excessive umbilical cord coiling confers risk of elevated nocturnal blood pressure and severe/early-onset preeclampsia. J Hypertens. 2019;37:187-196.

5. Debbie AL, Corrie M-W, Abigail F, et al. Cardiovascular biomarkers and vascular function during childhood in the offspring of mothers with hypertensive disorders of pregnancy: findings from the Avon Longitudinal Study of Parents and Children. Eur Heart J. 2012;33:335-345.

6. Byberg KK, Øymar K, Eide GE, Forman MR, Júlíusson PB. Exposure to preeclampsia in utero affects growth from birth to late childhood dependent on child's sex and severity of exposure: followup of a nested case-control study. PLoS One. 2017;12:e176627. doi:10.1371/journal.pone.0176627

7. Heikura U, Hartikainen AL, Nordstrom T, Pouta A, Taanila A, Jarvelin MR. Maternal hypertensive disorders during pregnancy and mild cognitive limitations in the offspring. Paediatr Perinat Epidemiol. 2013;27:188-198.

8. Rätsep MT, Hickman AF, Croy BA. Impacts of placental growth factor and preeclampsia on brain development, behavior, and cognition. Placenta. 2016;48:S40-D46. doi:10.1016/j.placenta. 2016.02.001

9. Rätsep MT, Hickman AF, Maser B, et al. Impact of preeclampsia on cognitive function in the offspring. Behav Brain Res. 2016;302:175-181. doi:10.1016/j.bbr.2016.01.030

10. Leversen KT, Sommerfelt K, Ronnestad A, et al. Prediction of neurodevelopmental and sensory outcome at 5 years in Norwegian children born extremely preterm. Pediatrics. 2011;127:e630-638. doi:10.1542/peds.2010-1001

11. Ehrenstein V, Rothman KJ, Pedersen L, Hatch EE, Sorensen HT. Pregnancy-associated hypertensive disorders and adult cognitive function among Danish conscripts. Am J Epidemiol. 2009;170 (8):1025-1031. doi:10.1093/aje/kwp223
12. Tuovinen S, Eriksson JG, Kajantie E, et al. Maternal hypertensive disorders in pregnancy and self-reported cognitive impairment of the offspring 70 years later: the Helsinki Birth Cohort Study. Am J Obstet Gynecol. 2013;208:200.e201-209. doi:10.1016/j.ajog.2012.12.017

13. Gudnadottir TA, Bateman BT, Hernadez-Diaz S, Luque-Fernandez MA, Valdimarsdottir U, Zoega H. Body mass index, smoking and hypertensive disorders during pregnancy: a population based case-control study. PLoS One. 2016;11:e0152187. doi:10.1371/journal.pone. 0152187

14. Maher GM, O'Keeffe GW, Kearney PM, et al. Association of hypertensive disorders of pregnancy with risk of neurodevelopmental disorders in offspring: a systematic review and meta-analysis. JAMA Psychiat. 2018;75:809-819. doi:10.1001/jamapsychiatry.2018.0854

15. Roberts JM. Endothelial dysfunction in preeclampsia. Semin Reprod Endocrinol. 1998;16(01):5-15. doi:10.1055/s-2007-1016248

16. Chau K, Hennessy A, Makris A. Placental growth factor and pre-eclampsia. J Hum Hypertens. 2017;31(12):782-786. doi:10.1038/jhh.2017.61

17. Rana S, Lemoine E, Granger JP, Karumanchi SA. Preeclampsia: pathophysiology, challenges, and perspectives. Circ Res. 2019;124:1094-1112.

18. Finkelstein JD, Martin JJ. Homocysteine. Int J Biochem Cell Biol. 2000;32(4):385-389. doi:10.1016/S1357-2725(99)00138-7

19. Dubchenko EA, Ivanov AV, Boiko AN, Spirina NN, Gusev EI, Kubatiev AA. Hyperhomocysteinemia and endothelial dysfunction in patients with cerebral vascular and autoimmune diseases. $Z h$ Nevrol Psikhiatr Im $S \quad S$ Korsakova. 2019;119:133-138. doi:10.17116/jnevro2019119111133

20. Lentz SR, Rodionov RN, Dayal S. Hyperhomocysteinemia, endothelial dysfunction, and cardiovascular risk: the potential role of ADMA. Atheroscler Suppl. 2003;4:61-65. doi:10.1016/S1567-5688(03) 00035-7

21. Musolino PL, Gong Y, Snyder JM, et al. Brain endothelial dysfunction in cerebral adrenoleukodystrophy. Brain. 2015;138:3206-3220.

22. Deng F, Wang S, Xu R, Yu W, Wang X, Zhang L. Endothelial microvesicles in hypoxic hypoxia diseases. $J$ Cell Mol Med. 2018;22(8):3708-3718. doi:10.1111/jcmm.13671

23. Picker JD, Coyle JT. Do maternal folate and homocysteine levels play a role in neurodevelopmental processes that increase risk for schizophrenia? Harv Rev Psychiatry. 2005;13:197-205. doi:10.1080/ 10673220500243372

24. Huseynova SA, Panakhova NF, Hajiyeva AS, Orujova PA, Mukhtarova SN, Agayeva GT. Endothelial dysfunction and developmental outcomes of very low birth weight newborns with hypoxic encephalopathy. J Pak Med Assoc. 2017;67:1857-1863.

25. Mol BWJ, Roberts CT, Thangaratinam S, Magee LA, de Groot CJM, Hofmeyr GJ. Pre-eclampsia. Lancet. 2016;387:999-1011. doi:10.1016/S0140-6736(15)00070-7

26. Li HT, Ye RW, Pei LJ, Ren AG, Zheng XY, Liu JM. Cesarean delivery on maternal request and childhood intelligence: a cohort study. Chin Med J (Engl). 2011;124:3982-3987.

27. Small HY, Currie GE, Delles C. Prostasin, proteases, and preeclampsia. J Hypertens. 2016;34:193-195. doi:10.1097/HJH.00 00000000000828

28. Duley L. The global impact of pre-eclampsia and eclampsia. Semin Perinatol. 2009;33:130-137.

29. Phipps EA, Thadhani R, Benzing T, Karumanchi SA. Pre-eclampsia: pathogenesis, novel diagnostics and therapies. Nat Rev Nephrol. 2019;15:275-289.

30. Chaiworapongsa T, Chaemsaithong P 1, Yeo L, Romero R. Preeclampsia part 1: current understanding of its pathophysiology. Nat Rev Nephrol. 2014;10:466-480.

31. Tomimatsu T, Mimura K, Endo M, Kumasawa K, Kimura T. Pathophysiology of preeclampsia: an angiogenic imbalance and long-lasting systemic vascular dysfunction. Hypertens Res. 2017;40:305-310. 
32. Welch GN, Upchurch GR Jr, Loscalzo J. Homocysteine, oxidative stress, and vascular disease. Hosp Pract. 1997;32:81-2,85, 88-92. doi:10.1080/21548331.1997.11443510

33. Sun LJ, Xu GF, Lv M, Zhou H, Huang HF, Luo Q. Predictive value of maternal serum biomarkers for preeclampsia and birth weight: a case-control study in Chinese pregnant women. J Womens Health (Larchmt). 2018;27:1519-1524. doi:10.1089/jwh.2017.6793

34. Maged AM, Saad H, Meshaal H, et al. Maternal serum homocysteine and uterine artery doppler as predictors of preeclampsia and poor placentation. Arch Gynecol Obstet. 2017;296:475-482. doi:10.1007/ s00404-017-4457-y

35. Bernardi FC, Felisberto F, Vuolo F, et al. Oxidative damage, inflammation, and toll-like receptor 4 pathway are increased in preeclamptic patients: a case-control study. Oxid Med Cell Longev. 2012;2012:636419. doi:10.1155/2012/636419

36. Tawakol A, Omland T, Gerhard M, Wu JT, Creager MA. Hyperhomocyst(e)inemia is associated with impaired endothelium-dependent vasodilation in humans. Circulation. 1997; 95:1119-1121. doi:10.1161/01.CIR.95.5.1119

37. Woo KS, Chook P, Lolin YI, et al. Hyperhomocyst(e)inemia is a risk factor for arterial endothelial dysfunction in humans. Circulation. 1997;96:2542-2544. doi:10.1161/01.CIR.96.8.2542

38. Massy ZA, Ceballos I, Chadefaux-Vekemens B, et al. Homocyst(e) ine, oxidative stress, and endothelium function in uremic patients. Kidney Int Suppl. 2001;78:S243-S245. doi:10.1046/j.15231755.2001.59780243.X

39. Zeng Y, Li M, Chen Y, Wang S. Homocysteine, endothelin-1 and nitric oxide in patients with hypertensive disorders complicating pregnancy. Int J Clin Exp Pathol. 2015;8:15275-15279.
40. Wang XC, Sun WT, Yu CM, et al. ER stress mediates homocysteine-induced endothelial dysfunction: modulation of IKCa and SKCa channels. Atherosclerosis. 2015;242:191-198. doi:10. 1016/j.atherosclerosis.2015.07.021

41. Sengupta A, Das U, Manna K, et al. An association study of severity of intellectual disability with peripheral biomarkers of disabled children in a rehabilitation home, Kolkata, India. Sci Rep. 2019;9:13652.

42. Smith AD, Refsum H. Homocysteine, B vitamins, and cognitive impairment. Annu Rev Nutr. 2016;36:211-239. doi:10.1146/annurevnutr-071715-050947

43. Sachdev PS. Homocysteine and brain atrophy. Prog Neuropsychopharmacol Biol Psychiatry. 2005;29:1152-1161. doi:10. 1016/j.pnpbp.2005.06.026

44. Murphy MM, Fernandez-Ballart JD, Molloy AM, Canals J. Moderately elevated maternal homocysteine at preconception is inversely associated with cognitive performance in children 4 months and 6 years after birth. Matern Child Nutr. 2017;13:e12289.

45. Dayan N, Kaur A, Elharram M, Rossi AM, Pilote L. Impact of preeclampsia on long-term cognitive function. Hypertension. 2018;72:1374-1380. doi:10.1161/HYPERTENSIONAHA.118.11320

46. Sverrisson FA, Bateman BT, Aspelund T, Skulason S, Zoega H. Preeclampsia and academic performance in children: a nationwide study from Iceland. PLoS One. 2018;13:e0207884.

47. Javadi EHS, Ghorbali F, Sarookhani M, Javadi A, Mashrabi O. The relationship between the level of homocysteine in mother's serum and the intensity of preeclampsia. Life Sci J. 2012;9:1247-1249.
Risk Management and Healthcare Policy

\section{Publish your work in this journal}

Risk Management and Healthcare Policy is an international, peerreviewed, open access journal focusing on all aspects of public health, policy, and preventative measures to promote good health and improve morbidity and mortality in the population. The journal welcomes submitted papers covering original research, basic science, clinical \& epidemiological studies, reviews and evaluations,

\section{Dovepress}

guidelines, expert opinion and commentary, case reports and extended reports. The manuscript management system is completely online and includes a very quick and fair peer-review system, which is all easy to use. Visit http://www.dovepress.com/testimonials.php to read real quotes from published authors. 